\title{
A mouse sperm decapacitation factor receptor is phosphatidylethanolamine-binding protein 1
}

\author{
Rachel Gibbons, Susan A Adeoya-Osiguwa and Lynn R Fraser \\ Reproduction and Rhythms Group, School of Biomedical Sciences, King's College London, Guy's Campus, \\ London SE1 1UL, UK
}

Correspondence should be addressed to L R Fraser; Email: lynn.fraser@kcl.ac.uk

\begin{abstract}
Capacitation is a pivotal event for mammalian spermatozoa, involving the loss of surface proteins known as decapacitation factors (DF) and consequent acquisition of fertilizing ability. Earlier studies showed that a mouse sperm DF binds to a receptor, DF-R, whose attachment to the sperm plasma membrane appears to involve a glycosylphosphatidylinositol (GPI) anchor. In the present study, purification and subsequent sequencing of DF-R has identified this $\sim 23 \mathrm{kDa}$ protein as phosphatidylethanolamine-binding protein 1 (PEBP 1). To obtain functional evidence that supports sequence homology data, purified recombinant PEBP 1 and PEBP 2 were evaluated for biological activity. While PEBP 1 was able to remove DF activity in solution at concentrations above $\sim 1 \mathrm{nmol} / \mathrm{I}$, PEBP 2 was ineffective, even at $600 \mathrm{nmol} / \mathrm{l}$; this confirmed that DF-R is PEBP 1 . Anti-PEBP 1 antiserum recognized recombinant PEBP 1 and a $\sim 23 \mathrm{kDa}$ protein in both mouse and human sperm lysates. Immunolocalization studies revealed that DF-R/PEBP 1 is located on the acrosomal cap, the post-acrosomal region and the flagellum of both mouse and human spermatozoa, with epitope accessibility being capacitation state-dependent and reversible. Treatment of cells with a phospholipase able to cleave GPI anchors essentially abolished immunostaining, thus confirming the extracellular location of DF-R/PEBP 1. We suggest that DF-R/PEBP 1 plays its fundamental role in capacitation by causing alterations in the sperm plasma membrane in both head and flagellum, with functional consequences for membrane-associated proteins. Obtaining more detail about DF $\leftrightarrow$ DF-R interactions could lead to useful applications in both fertility treatments and new contraceptive approaches.

Reproduction (2005) 130 497-508
\end{abstract}

\section{Introduction}

When released from the male reproductive tract, mammalian spermatozoa are non-fertilizing and require a further, species-dependent, period of hours during which they complete maturation and acquire the capacity to fertilize an oocyte, termed capacitation (Austin 1952). Only fully capacitated cells can interact with unfertilized oocytes and undergo the acrosome reaction, triggered by specific molecular interactions between the spermatozoon and the zona pellucida, prior to fertilizing the oocyte. The events that comprise capacitation are complex and still poorly understood (de Lamirande et al. 1997), but a crucial component is the loss of proteins from the sperm surface. If these proteins are added to capacitated, fertile suspensions spermatozoa revert to the uncapacitated, non-fertilizing state but in time can re-capacitate; thus, capacitation is reversible. These surface proteins have been given the general term 'decapacitation factors' or DF (Bedford \& Chang 1962). In some species, DF is present in seminal plasma and only contacts spermatozoa at ejaculation but, in others, DF is present on cells prior to release.

One such DF, found on epididymal mouse spermatozoa, has been studied for over two decades. Its removal from uncapacitated cells by gentle centrifugation results in highly fertile cells, while its addition to capacitated suspensions rapidly, but reversibly, inhibits fertilizing ability (Fraser 1984). This DF is a protein of $\sim 40 \mathrm{kDa}$, stable when boiled, and it appears to have carbohydrate residues that contribute to biological activity (Fraser et al. 1990). The binding of DF to spermatozoa results in stimulation of a calmodulin-sensitive $\mathrm{Ca}^{2+}$-ATPase and so helps to maintain low intracellular $\mathrm{Ca}^{2+}$ levels; immunolocalization studies have found $\mathrm{Ca}^{2+}$-ATPase to be located mainly in the post-acrosomal (posterior) region of the sperm head (Adeoya-Osiguwa \& Fraser 1996). During capacitation, however, DF is lost from the sperm surface and somehow inactivated; thus its effect on the $\mathrm{Ca}^{2+}$ pump declines, allowing intracellular $\mathrm{Ca}^{2+}$ to rise and so stimulate 
changes needed to make spermatozoa functionally competent.

Initially the DF was thought to bind directly to the sperm plasma membrane but this proved to be incorrect. Treating suspensions with phosphatidylinositol-specific phospholipase C (PI-PLC) to cleave glycosylphosphatidylinositol (GPI) anchors resulted in significant loss of DF and consequent advancement to the capacitated state, as determined using chlortetracycline (CTC) fluorescence. However, addition of DF to PI-PLC-treated suspensions caused no reversion to the uncapacitated state, suggesting that DF could no longer bind to these cells (Fraser 1998). Thus it is not the DF that has a GPI anchor but some other molecule, to which DF binds; this latter molecule has been called the DF receptor (DF-R). Further experiments revealed that DF has fucose moieties and DF-R has complementary fucose-binding sites, the latter being located particularly in the post-acrosomal region of the sperm head where $\mathrm{Ca}^{2+}$-ATPase has also been localized (Fraser 1998). Addition of exogenous fucose to uncapacitated spermatozoa quickly displaces endogenous DF, resulting in highly fertile suspensions and so demonstrating the functional importance of the fucose residues.

These molecules and their mechanism of action are not restricted to mouse spermatozoa. Evidence from many mammalian species has supported the involvement of a $\mathrm{Ca}^{2+}$-ATPase in capacitation and the mouse sperm DF has been shown to act on human, as well as mouse, spermatozoa. Addition of mouse DF to capacitated human sperm suspensions for $1 \mathrm{~h}$ caused a significant reversion to the uncapacitated state, determined by CTC (DasGupta et al. 1994). Furthermore, the addition of exogenous fucose to human sperm suspensions resulted in acceleration of capacitation (Fraser \& Osiguwa 2004), suggesting the presence in human spermatozoa of proteins similar to mouse DF and DF-R. The present study was designed to attempt purification and subsequent evaluation of mouse sperm DF-R.

\section{Materials and Methods}

All animals used in this study were maintained in Home Office-approved facilities.

\section{Media \\ The standard medium used was a modified Tyrode's med- ium (Fraser 1993) containing $1.8 \mathrm{mmol} / \mathrm{l} \quad \mathrm{CaCl}_{2}$ and $4 \mathrm{mg} / \mathrm{ml}$ bovine serum albumin (BSA; Sigma, Poole, UK). In some experiments, a modified medium lacking $\mathrm{CaCl}_{2}$ and containing $4 \mathrm{mg} / \mathrm{ml}$ polyvinyl alcohol (PVA; Sigma) in place of BSA was used (- $\mathrm{Ca}^{2+}+$ PVA).}

\section{Preparation of crude DF-R}

The contents of cauda epididymides from 20 mature TO male mice (Harlan, Bicester, Oxon, UK) were released into PVA-supplemented medium (1 ml per pair of epididymides). Spermatozoa were incubated on a warming tray at $37^{\circ} \mathrm{C}$ for $5 \mathrm{~min}$ to allow dispersal, then transferred to a capped plastic vial and incubated at $37^{\circ} \mathrm{C}$ for a further $25 \mathrm{~min}$; prior to all incubations, suspensions were gassed using $5 \% \mathrm{O}_{2}, 5 \% \mathrm{CO}_{2}, 90 \% \mathrm{~N}_{2}$. The suspension was centrifuged at $4500 \mathrm{~g}$ for $8 \mathrm{~min}$ and the cell-free supernatant containing the DF was removed. The pelleted cells were resuspended in the same volume of medium and pooled; 75 units PI-PLC (Sigma) were added and the suspension was incubated at $37^{\circ} \mathrm{C}$ for $1 \mathrm{~h}$. PI-PLCs are virulence factors produced by many pathogenic bacteria and are known to act by cleaving GPI-anchored proteins (Zenewicz et al. 2005). Sigma's PI-PLC, purified from the pathogenic bacterium Bacillus cereus, has a minimum of 1000 units PI-PLC activity/mg protein, with negligible PLC contamination that might cleave other types of lipids (no more than 2 units PLC activity/mg protein); it also contains EDTA to chelate zinc, a cofactor for PLC, in order to inactivate any PLC. Therefore it is unlikely that enzyme treatment of spermatozoa caused non-specific cleavage of more general lipid linkages. After centrifuging the suspension at $4500 \mathrm{~g}$ for $8 \mathrm{~min}$, the cell-free supernatant containing DF-R was collected, lyophilized and stored at $-20^{\circ} \mathrm{C}$.

\section{Partial purification of DF-R by immobilized fucose affinity chromatography}

Lyophilized crude DF-R was reconstituted in $4 \mathrm{ml}$ deionized water $(200 \mu \mathrm{l}$ per male originally used) and then subjected to affinity chromatography at $4{ }^{\circ} \mathrm{C}$ on L-fucose cross-linked $6 \%$ beaded agarose (Sigma). The column $(1.5 \mathrm{~cm} \times 10 \mathrm{~cm})$ had been freshly equilibrated with $50 \mathrm{mmol} / \mathrm{l}$ phosphate-buffered saline (PBS) overnight. The crude DF-R was loaded onto the column with PBS at a flow rate of $\sim 8 \mathrm{ml} / \mathrm{h}$; this was then increased and maintained at $\sim 17 \mathrm{ml} / \mathrm{h}$; typically $80 \times 1 \mathrm{ml}$ fractions were collected. Elution buffer $(500 \mathrm{mmol} / \mathrm{l} \mathrm{NaCl}+50 \mathrm{mmol} / \mathrm{l}$ fucose in $200 \mathrm{mmol} / \mathrm{l}$ phosphate buffer) was added at approximately fraction 45 . A representative range of these fractions was subjected to SDS-PAGE and protein assay; samples were denatured by boiling for $7 \mathrm{~min}$ in loading buffer $(6 \%$ SDS and $150 \mathrm{mmol} / \mathrm{l}$ dithiothreitol) and resolved on 12\% SDS-PAGE (Protogel; National Diagnostics, Hull, Humberside, UK). Those containing DF-R activity were lyophilized, reconstituted in $4 \mathrm{ml}$ deionized water and dialysed against 250 volumes deionized water (ten changes of at least $2 \mathrm{~h}$ each). The dialysed samples were then lyophilized overnight.

\section{CTC analysis of DF-R activity in partially purified preparations}

Sperm suspension preparation was carried out as described for crude DF-R preparation, except that the spermatozoa were released into $0.8 \mathrm{ml}$ standard medium $\left(+\mathrm{Ca}^{2+}+\mathrm{BSA}\right)$ per pair of epididymides. Following dispersal of the cells, an aliquot of sperm suspension was 
removed and centrifuged at $4500 \mathrm{~g}$ for $8 \mathrm{~min}$; the supernatant, containing crude DF, was kept on ice until just before use. The remainder of the sperm suspension was filtered through short columns of Sephadex G-25 (medium grade; Amersham Biosciences, Little Chalfont, Bucks, UK) to remove immotile cells. The filtered cells were gassed, incubated for $25 \mathrm{~min}$ at $37^{\circ} \mathrm{C}$ and divided into $150 \mu \mathrm{l}$ aliquots. Suspensions were then centrifuged at $1100 \mathrm{~g}$ to remove endogenous DF, supernatant was removed and cells were resuspended in $100 \mu \mathrm{l}$ of the relevant treatment solutions detailed below; the final sperm concentration was $\sim 1-2 \times 10^{6} / \mathrm{ml}$. After a further 10-min incubation at $37^{\circ} \mathrm{C}$, the capacitation state of the cells was assessed using CTC. Slide preparation and assessment were carried out as described previously (Fraser 1998). In each treatment, 100 cells were assessed for one of the three main CTC staining patterns: F, with fluorescence over the entire head, characteristic of uncapacitated acrosome-intact cells; B, with a fluorescence-free band in the post-acrosomal region, characteristic of capacitated acrosome-intact cells; AR, with dull or absent fluorescence over the entire head, characteristic of capacitated acrosome-reacted cells.

\section{Preparation of low molecular weight (LMW) DF-R fraction}

Lyophilized partially purified DF-R samples were reconstituted in $400 \mu \mathrm{l}$ deionised water and centrifuged through an Ultrafree-MC centrifugal filter unit (Millipore, Watford, Herts, UK) with a nominal molecular weight limit of $30 \mathrm{kDa}$, for $20 \mathrm{~min}$ at $2000 \mathrm{~g}$. The resulting high molecular weight $(\mathrm{HMW})$ and $\mathrm{LMW}$ fractions were lyophilized and stored at $-20^{\circ} \mathrm{C}$.

\section{Protein sequencing}

Sequencing by liquid chromatographic mass spectrometry (LC/MS/MS) was carried out on the single Coomassiestained SDS-PAGE protein band of $\sim 23 \mathrm{kDa}$ found in the LMW fraction of the partially purified DF-R preparation. The mass spectral data were processed into peak lists and searched against the Swiss Prot database (www.ebi.ac. uk/swissprot) using Mascot software (Matrix Science, London, UK).

\section{pebp 1 and pebp 2 transcript detection in various tissues}

Total RNA was extracted from mouse brain, testis and epididymis using an RNeasy Maxikit (Qiagen, Crawley, W Sussex, UK) according to the manufacturer's protocol. cDNA was then synthesized for each tissue using the Thermoscript RT-PCR System (Invitrogen, Paisley, Strathclyde, UK) following the manufacturer's protocol. PCR was carried out to amplify complete pebp 1 coding sequence plus Ndel and Hind III restriction enzyme digestion sites to aid in cloning, with and without the addition of sequence coding for a $6 \times$ histidine (His) tag to aid in purifying the protein once the sequence had been cloned and expressed in bacteria. This was achieved by mixing $1 \mu \mathrm{l}$ cDNA with $200 \mathrm{nmol} / \mathrm{l}$ of each specific primer ( pebp 1-His forward primer 5'-ATATACATATGGCCGCCGACATCAGCC- ${ }^{\prime}$, pebp $1+$ His forward primer 5'-ATATACATATGCATCATCACCATCATCACGCCGCCGACATCAGCC-3', pebp $1 \pm$ His reverse primer $5^{\prime}$-ACGACAAGCTTCCTACTTCCCTGACAGCTGC-3'), $200 \mathrm{nmol} / \mathrm{l}$ dNTP mix and 2.6U Expand enzyme mix in $50 \mu \mathrm{l} 1 \times$ reaction buffer (Roche Diagnostics, Basel, Switzerland). The samples then underwent the following PCR cycles in a DYAD Peltier thermal cycler (MJ Technologies, Coalville, Leics, UK): 1 cycle of $2 \mathrm{~min}$ at $94^{\circ} \mathrm{C} ; 10$ cycles of $10 \mathrm{~s}$ at $96^{\circ} \mathrm{C}, 30$ s at $55^{\circ} \mathrm{C}, 1 \mathrm{~min}$ at $68^{\circ} \mathrm{C} ; 25$ cycles of $10 \mathrm{~s}$ at $96^{\circ} \mathrm{C}$, $30 \mathrm{~s}$ at $55^{\circ} \mathrm{C}, 1 \mathrm{~min}+5 \mathrm{~s} / \mathrm{cycle}$ at $68^{\circ} \mathrm{C} ; 1$ cycle of $7 \mathrm{~min}$ at $68^{\circ} \mathrm{C}$, followed by continuous incubation at $4^{\circ} \mathrm{C}$. The amplification of pebp 2 coding sequence plus Ndel and HindllI restriction enzyme digestion sites, with and without the addition of sequence coding for a His tag, used the same reaction components and PCR cycles as that described for pebp 1 but required two rounds of nested PCR (pebp 2 first round forward primer 5'-GACTCCAGCCTCTGACC-3', pebp 2 first round reverse primer $5^{\prime}$-TGGCCATGATCATCAAGTGATC-3', pebp 2-His second round forward primer $5^{\prime}$ ATATACATATGCCTACAGACATGAGCATGTGGA- $3^{\prime}$, pebp $2+$ His second round forward primer $5^{\prime}$-ATATACATATGCATCATCACCATCATCACCCTACAGACATGAGCATGTGA-3', pebp $2 \pm$ His second round reverse primer $5^{\prime}$-ACGACAAGCTTCAAGTGATCCCCCTATTTCC- $3^{\prime}$ ). Samples were subjected to electrophoresis on a $1.2 \%$ agarose gel prepared in TAE buffer $(40 \mathrm{mmol} / \mathrm{l}$ Tris-acetate and $1 \mathrm{mmol} / \mathrm{I} \mathrm{EDTA}, \mathrm{pH}$ $8.0)$ at $100 \mathrm{~V}$ for approximately $35 \mathrm{~min}$.

\section{Production and purification of recombinant PEBP 1 and PEBP 2 proteins}

Purified pebp 1 and pebp $2 \pm$ His PCR products were cloned first into TOPO cloning vector in TOP 10 cells (Invitrogen) and subsequently into the expression vector used in this investigation, pET 21a (Novagen, Nottingham, UK), according to the manufacturers' protocols. The expression vectors containing the inserted sequences were confirmed by DNA sequencing.

The expression host used in this work, Escherichia coli BL21 (DE3) pLysS (Novagen), was transformed with the pET 21 a vectors containing pebp 1 and pebp $2 \pm$ His according to the manufacturer's protocol for chemical transformation, and propagated overnight in LB broth (Sigma) plus ampicillin $(100 \mu \mathrm{g} / \mathrm{ml})$ at $37^{\circ} \mathrm{C}$. The culture was then used to inoculate $(2 \%, v / v) 100 \mathrm{ml}$ LB broth (sigma) plus ampicillin $(100 \mu \mathrm{g} / \mathrm{ml})$ for over-expression of proteins. Cultures were incubated at $37^{\circ} \mathrm{C}$ with shaking and, after $\sim 3 \mathrm{~h}$, overexpression was induced by the addition of $0.4 \mathrm{mmol} / \mathrm{l}$ isopropyl $\beta$-D-thiogalactopyranosidase (Sigma). Cells were harvested at $5 \mathrm{~h}$ post-induction by centrifugation at $5000 \mathrm{~g}$ for $15 \mathrm{~min}$ and stored at $-80^{\circ} \mathrm{C}$ until required.

Purification of PEBP $1+$ His and PEBP $2+$ His proteins was achieved using nickel-nitrilotriacetic acid (Ni-NTA) 
affinity chromatography (Invitrogen) according to the manufacturer's protocol. Briefly, $50 \mathrm{ml}$ pelleted cells were resuspended in $8 \mathrm{ml}$ native binding buffer $(50 \mathrm{mmol} / \mathrm{l}$ $\mathrm{NaH}_{2} \mathrm{PO}_{4}, 500 \mathrm{mmol} / \mathrm{l} \mathrm{NaCl}$ and $10 \mathrm{mmol} / \mathrm{l}$ imidazole) and sonicated. Insoluble material was removed by centrifugation at $3000 \mathrm{~g}$ for $15 \mathrm{~min}$. Cell-free supernatant containing PEBP $1+$ His or PEBP $2+$ His was removed and affinity chromatography was carried out as described for DF-R except that the beaded agarose used was coated with Ni-NTA, the wash buffer contained $50 \mathrm{mmol} / \mathrm{l}$ $\mathrm{NaH}_{2} \mathrm{PO}_{4}, 500 \mathrm{mmol} / \mathrm{l} \mathrm{NaCl}$ and $20 \mathrm{mmol} / \mathrm{l}$ imidazole and the elution buffer used contained $50 \mathrm{mmol} / \mathrm{l}$ $\mathrm{NaH}_{2} \mathrm{PO}_{4}, 500 \mathrm{mmol} / \mathrm{l} \mathrm{NaCl}$ and $250 \mathrm{mmol} / \mathrm{l}$ imidazole. SDS-PAGE was carried out on a range of fractions and those containing PEBP $1+$ His or PEBP $2+$ His were dialysed as described for DF-R.

\section{Anti-PEBP 1 antibody production and Western blotting}

Antigenic prediction, peptide synthesis/purification and antibody production/purification were carried out by Cambridge Research Biochemicals (Billingham, Cleveland, UK). Briefly, a synthetic peptide (EWDDYVPKLYEQLSGGC) was prepared, based on predicted differences in structure between PEBP 1 and PEBP 2 proteins at the carboxyl terminus, and purified using HPLC. The peptide was then conjugated to keyhole limpet haemocyanin and used to immunize two New Zealand white rabbits over an 85-day protocol, to generate high titre anti-PEBP 1 serum. Specific anti-PEBP 1 antibodies were purified by affinity chromatography against the synthetic peptide.

Western blotting was carried out against the purified PEBP $1+$ His and PEBP $2+$ His with an anti-His tag antibody to confirm the presence of both proteins and then with anti-PEBP 1 to confirm specificity for PEBP 1. Proteins were resolved using SDS-PAGE and transferred to PVDF membrane (Amersham Biosciences). Membranes were blocked in 5\% milk in Tris-buffered saline and $0.1 \%$ Tween (TBST) for $1 \mathrm{~h}$ at room temperature and then incubated overnight at $4{ }^{\circ} \mathrm{C}$ in either 1:3000 mouse anti-His tag antibody (Amersham Biosciences) or 1:10000 rabbit anti-PEBP 1 polyclonal antibody in $0.5 \%$ milk in TBST. The membranes were washed (three times for $5 \mathrm{~min}$ each in TBST) and then incubated at room temperature for $1 \mathrm{~h}$ in peroxidase-linked secondary antibody (1:250 000 sheep anti-mouse IgG or 1:100000 donkey anti-rabbit IgG in $0.5 \%$ milk in TBST). After further washing (six times for 5 min each in TBST), proteins were detected using an ECLPlus Western blotting detection system on ECL Hyperfilm (Amersham Biosciences).

Once the antiserum specificity had been confirmed, it was used to detect PEBP 1 in both mouse and human sperm lysates prepared by freeze-thawing mature epididymal and ejaculated sperm suspensions respectively, in liquid $\mathrm{N}_{2}$, pelleting the cells and resuspending in PBS containing $40 \mu \mathrm{g} / \mathrm{ml}$ trypsin inhibitor and $0.2 \mu \mathrm{g} / \mathrm{ml}$ leupeptin. SDS-PAGE and immunoblotting were carried out as above except that anti-PEBP 1 was used at 1:1000 and the anti-rabbit IgG was used at 1:10000.

\section{Immunocytochemical localization of DF-R/PEBP 1}

To look for possible capacitation state-dependent differences, several different evaluations were carried out on both uncapacitated and capacitated mouse and human sperm suspensions. Both unpermeabilized and permeabilized (mixed 1:1 with 4\% (w/v) sucrose to help stabilize cellular fine structure, then permeabilized by freezing in liquid $\mathrm{N}_{2}$, thawed and fixed with $2.5 \%(\mathrm{w} / \mathrm{v})$ paraformaldehyde) preparations were evaluated in pilot experiments. While the same distribution was seen in both preparations, the permeabilized ones gave better resolution and so this approach was used in all subsequent preparations.

Mouse sperm suspensions were prepared as described for CTC analysis. Uncapacitated samples in $-\mathrm{Ca}^{2+}+$ PVA medium were prepared either immediately for immunocytochemistry as described by Adeoya-Osiguwa \& Fraser (2002) or were incubated in $-\mathrm{Ca}^{2+}+$ PVA medium plus 5 units PI-PLC for $1 \mathrm{~h}$ at $37^{\circ} \mathrm{C}$ and then prepared for immunocytochemistry. DF-depleted samples were prepared in $+\mathrm{Ca}^{2+}+\mathrm{BSA}$ control medium, resuspended in (1) control medium for $10 \mathrm{~min}$ at $37^{\circ} \mathrm{C}$, (2) crude DF for $10 \mathrm{~min}$ at $37^{\circ} \mathrm{C}$ or (3) control medium containing 5 units PI-PLC for $1 \mathrm{~h}$ at $37^{\circ} \mathrm{C}$ and then prepared for immunocytochemistry. Naturally capacitated samples, obtained by incubating cells in $+\mathrm{Ca}^{2+}+\mathrm{BSA}$ medium in $5 \% \mathrm{O}_{2}, 5 \% \mathrm{CO}_{2}, 90 \% \mathrm{~N}_{2}$ for $2 \mathrm{~h}$, were then (1) immediately prepared for immunocytochemistry, (2) centrifuged at $1100 \mathrm{~g}$ and resuspended in crude DF for $10 \mathrm{~min}$ at $37^{\circ} \mathrm{C}$ or (3) had 5 units PI-PLC added for $1 \mathrm{~h}$ at $37^{\circ} \mathrm{C}$ and then prepared for immunocytochemistry. Slides were prepared, treated and examined as described by Adeoya-Osiguwa \& Fraser (2002) except that the cells were left to bind to the poly-L-lysine-coated slides for $10 \mathrm{~min}$, the anti-PEBP 1 primary antibody was used at a 1:25 dilution and the anti-rabbit IgG-biotinylated secondary antibody was used at a dilution of 1:100.

Human semen samples were obtained from normal adult males (with approval from the King's College Research Ethics Committee). Human sperm suspension preparation was carried out using discontinuous Percoll density gradient centrifugation; washed cells were resuspended in Earle's medium with added penicillin and human serum albumin (Fraser \& Osiguwa 2004); gentle centrifugation of human spermatozoa does not remove their DF. Uncapacitated samples were prepared either immediately for immunocytochemistry at a concentration of $4 \times 10^{7}$ cells/ml or incubated for $1 \mathrm{~h}$ at $37^{\circ} \mathrm{C}$ with 5 units PI-PLC at $5 \times 10^{6}$ cells $/ \mathrm{ml}$, then centrifuged at $700 \mathrm{~g}$, resuspended to $4 \times 10^{7}$ cells $/ \mathrm{ml}$ and prepared for immunocytochemistry. To obtain capacitated samples, suspensions at a concentration of $5 \times 10^{6}$ cells $/ \mathrm{ml}$ were incubated for $24 \mathrm{~h}$ at $37^{\circ} \mathrm{C}$ in $5 \% \mathrm{O}_{2}, 5 \% \mathrm{CO}_{2}, 90 \% \mathrm{~N}_{2}$. After $23 \mathrm{~h}$ of incubation, 5 units PI-PLC were added to half the suspension and at $24 \mathrm{~h}$ 
both treated and untreated samples were centrifuged at $700 \mathrm{~g}$, resuspended to $4 \times 10^{7}$ cell $\mathrm{s} / \mathrm{ml}$ and prepared for immunocytochemistry. Slides were prepared, treated and examined as described for mouse samples except that cells were left to bind to slides for $20 \mathrm{~min}$, the anti-PEBP 1 antibody was used at a dilution of 1:50 and the secondary antibody was used at a dilution of 1:200. Since CTC analysis has already shown that addition of mouse DF to similarly capacitated human spermatozoa causes reversion to the uncapacitated state (DasGupta et al. 1994), it was decided not to include this treatment in the present study.

\section{Statistical analysis}

Analysis of CTC data used Cochran's modification of the $\chi^{2}$ test (Snedecor \& Cochran 1980) which compares responses within each replicate and then sums the values. The difference between the experimental and control values in each replicate must be sufficiently large and the responses must be consistent in order to have a significant difference.

\section{Results}

\section{Fucose-based affinity purification of DF-R}

When the crude DF-R preparation was subjected to affinity chromatography on L-fucose-beaded agarose, typically two identifiable protein peaks were present (Fig. 1A), a large first peak corresponding to the void volume (see Fig. 1B) and, following addition of elution buffer, a small second peak. SDS-PAGE carried out on the latter fractions indicated the presence of a protein of $\sim 23 \mathrm{kDa}$ plus several proteins of considerably higher molecular weights

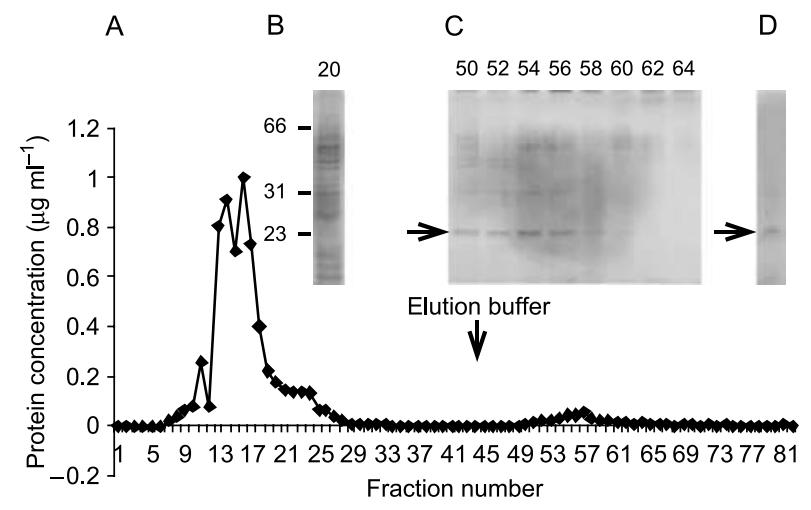

Figure 1 Fucose-based affinity purification of DF-R. (A) A crude DF-R preparation was applied to a column packed with agarose beads coated in L-fucose, using PBS as the equilibration buffer. At fraction 45 , elution buffer containing $500 \mathrm{mM} \mathrm{NaCl}$ and $50 \mathrm{mM}$ fucose was added; this figure shows the protein concentration profile obtained. Fractions 50-62 were pooled and evaluated further. (B) A silverstained SDS-PAGE gel shows proteins found in fraction 20 of the void volume; selected molecular weight marker positions are indicated on the left. (C) A silver-stained SDS-PAGE gel of selected eluted fractions shows a prominent protein of $\sim 23 \mathrm{kDa}$ (arrow). (D) The Coomassiestained gel shows the single protein band at $\sim 23 \mathrm{kDa}$ (arrow) that was subsequently sequenced.
(Fig. 1C). Previous work had suggested that DF-R might be $<30 \mathrm{kDa}$ (Rakha et al. 2000) and so subsequent work focused on this protein.

\section{Effect of partially purified DF-R on capacitation}

Fractions containing the suspected DF-R were pooled, dialysed and tested for biological activity using the CTC fluorescence assay. We have shown in numerous studies that suspensions having a relatively high proportion of cells with the CTC F pattern (uncapacitated, acrosome intact) are poorly fertilizing, while those with a relatively high proportion of B pattern cells (capacitated, acrosome intact) are highly fertile (Fraser et al. 1990, Fraser 1998, Fraser \& Osiguwa 2004). For example, incubation of uncapacitated suspensions for a short time in the presence of exogenous fucose, which displaces endogenous DF, caused a marked shift from $\mathrm{F}$ pattern to B pattern cells, compared with untreated controls, suggesting that capacitation was accelerated by the fucose treatment. Consistent with these results, fucose-treated mouse sperm suspensions were significantly more fertile in vitro than untreated controls (Fraser 1998).

Sperm suspensions were prepared and, after dispersal, an aliquot was removed and centrifuged to prepare crude DF. The remaining suspension was incubated at $37^{\circ} \mathrm{C}$ for a further $25 \mathrm{~min}$ and then divided into $150 \mu \mathrm{l}$ aliquots and centrifuged to remove endogenous DF; an uncentrifuged aliquot served as the uncapacitated control. Pellets were resuspended in $100 \mu \mathrm{l}$ of either control medium, crude DF or crude DF plus partially purified DF-R sample $(\sim 0.2 \mu \mathrm{g} / \mathrm{ml}$ total protein); $10 \mathrm{~min}$ after resuspension, spermatozoa were stained with CTC to assess their capacitation state $(n=3)$. Removal of DF by centrifugation resulted in accelerated capacitation in control cells (Fig. 2), with the majority of cells displaying the capacitated B pattern of fluorescence. The addition of crude DF to washed cells caused a reversion to the decapacitated state, as shown by a significant increase $(P<0.01)$ in the proportion of uncapacitated $\mathrm{F}$ pattern cells and a corresponding significant decrease $(P<0.01)$ in capacitated $\mathrm{B}$ pattern cells, consistent with earlier studies (Fraser 1998). However, when cells were resuspended in crude DF plus partially purified DF-R, the distribution of CTC patterns was essentially that seen in the centrifuged control cells, with a majority of capacitated B pattern cells. Thus DF-R activity was present in the preparation and interfered with responses to the crude DF by binding to DF in solution, leaving little or no unbound DF available to bind to endogenous DF-R present on spermatozoa.

\section{Evaluation of $H M W$ and LMW fractions for DF-R activity}

To confirm that the $23 \mathrm{kDa}$ protein was responsible for the activity detected, the sample was centrifuged on a filter column with a molecular weight exclusion limit of $30 \mathrm{kDa}$ to remove the HMW proteins that had been detected 


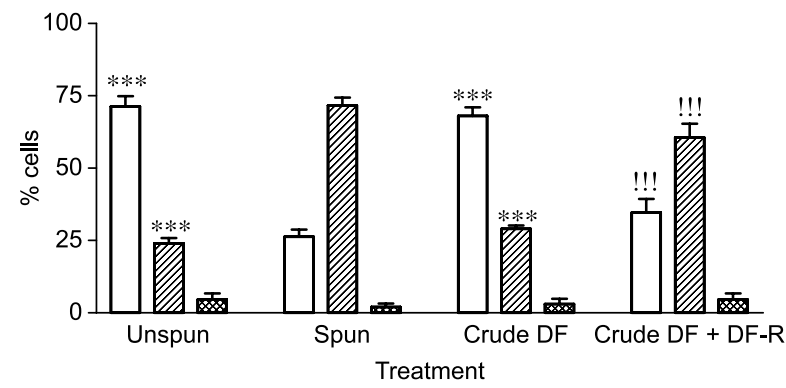

Figure 2 Biological activity of partially purified DF-R. Mouse sperm suspensions were incubated for $25 \mathrm{~min}$ to allow capacitation to begin, centrifuged to remove endogenous DF, then resuspended in media of different compositions with respect to crude DF and partially purified DF-R (unspun = uncapacitated cells), spun, DFdepleted control, crude DF and crude DF + DF-R) for $10 \mathrm{~min}$. Cells were stained with CTC, fixed and assessed for any differences in the distribution of fluorescence patterns. Data are presented as \% cells (means \pm S.E.; $n=3$ ) expressing the $\mathrm{F}$ pattern (open bars), the B pattern (hatched bars) and the AR pattern (cross-hatched bars) of CTC fluorescence. ${ }^{* * *} P<0.01$ compared with spun (DF-depleted control) suspensions and !!! $P<0.01$ compared with crude DF-treated suspensions.

following SDS-PAGE. SDS-PAGE plus silver staining of the HMW fraction revealed that it contained both HMW and LMW proteins; the most abundant protein was $\sim 23 \mathrm{kDa}$, indicating that not all the small proteins had passed through the filter. However, the LMW DF-R fraction showed only one visible protein of $\sim 23 \mathrm{kDa}$. The biological activity of both fractions was assessed as described above, with prepared, centrifuged sperm suspensions being resuspended in control medium, crude DF or crude DF plus LMW DF-R or HMW DF-R fractions. CTC results $(n=3)$ were essentially the same as those obtained with the unfractionated purified DF-R sample (data not shown). Since the only protein common to both fractions was the $23 \mathrm{kDa}$ protein, the protein in the LMW fraction (Fig. 1D) was sequenced using LC/MS/MS.

\section{LC/MS/MS sequence data}

LC/MS/MS analysis revealed very high homology between the amino acid sequence data generated for DF-R and that for PEBP 1 (Fig. 3). When aligned with the published sequence for PEBP 1, there was 100\% homology for the three peptide regions detected in our analysis (comprising $76 \%$ of the full PEBP 1 sequence), while three peptide fragments were missing. Several other studies on PEBP 1 have reported that the protein is $\mathrm{NH}_{2}$-terminally blocked (e.g. Jones \& Hall 1991, Pryor et al. 1994), which could account for the 'missing' first 26 amino acids. Based on these results, we have concluded that DF-R is PEBP 1. To test this hypothesis further, it was necessary to produce and purify recombinant PEBP 1 and its family member, PEBP 2, and then evaluate them for biological activity.

\section{pebp 1 and pebp 2 transcript detection}

Following reverse transcription and PCR, DNA fragments of approximately the expected sizes (573 bp and $591 \mathrm{bp}$ ) were consistently generated for pebp $1 \pm$ His in brain, testis and epididymis samples using cDNA primed with either oligo (dT) ${ }_{20}$ or random hexamers (Fig. 4). However, in order to generate DNA fragments for pebp $2 \pm \mathrm{His}$ it was necessary to use nested PCR; a first round product was only generated from the oligo $(\mathrm{dT})_{20}$ testis cDNA (data not shown) and this was used to generate both the pebp $2 \pm$ His products (585 bp and $603 \mathrm{bp}$ ).

\section{Functional evaluation of purified recombinant PEBP 1 and PEBP 2}

The effect of purified recombinant PEBP $1+$ His and PEBP $2+$ His proteins upon the capacitation state of sperm was tested using CTC $(n=3)$. Sperm suspensions were prepared as previously described and resuspended in control medium, crude DF or crude DF plus varying concentrations of PEBP 1 or PEBP 2. When PEBP 1 was tested using concentrations ranging from 0.7 to $200 \mathrm{nmol} / \mathrm{l}$, a
PEBP1 MAADISQWAGPLCLQEVDEPPQHALRVDYGVTVDELGKVLTPTQVMNRPSSISWDGLDP
DF-R - - VDYAGVTVDELGVLTPTQVMNRPSSISWDGLDP

PEBP1 GKLYTLVLTDPDAPSRKDPKFREWHHFLVVNMKGNDISSGTVLSDYVGSGPPSGTGLHRY DF-R GKLYTLVLTDPDAPSRKDP__________KGNDISSGTVLSDYVGSGPPSGTGLHRY

PEBP1 VWLVYEQEQPLSCDEPILSNKSGDNRGKFKVETFRKKYNLGAPVAGTCYQAEWDDYVPKL DF-R VWLVYEQEQPLSCDEPILSNKSG-_-KFKVETFRKKYNLGAPVAGTCYQAEWDDYVPKL

PEBP1 YEQLSGK

DF-R YEQLSGK

Figure 3 Comparison of the predicted amino acid sequence of PEBP 1 (accession number P70296) and the amino acid sequence data generated for purified DF-R (protein seen in Fig. 1D) using LC/MS/MS. The asterisk indicates an identical amino acid in the DF-R sequence and — indicate missing amino acids. 
A

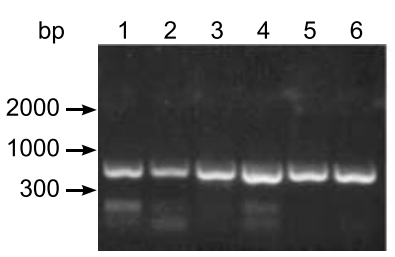

B

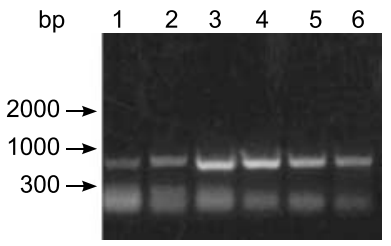

Figure 4 PCR detection of pebp 1 transcripts in brain, testis and epididymis. Ethidium bromide staining was used to visualize the complete (A) pebp 1-His PCR product and (B) pebp 1+ His PCR product from oligo $d(T)_{20}$-primed brain cDNA (lane 1), random hexamerprimed brain cDNA (lane 2), oligo d(T) ${ }_{20}$-primed testis cDNA (lane 3 ), random hexamer-primed testis cDNA (lane 4), oligo d(T) 20 -primed epididymis cDNA (lane 5) and random hexamer-primed epididymis cDNA (lane 6), run on a 1.2\% agarose gel. Base pair standards are indicated on the left of both gels.

maximal response was obtained with $7 \mathrm{nmol} / \mathrm{l}$ and higher (results are not shown for concentrations greater than $20 \mathrm{nmol} / \mathrm{l})$; there were significantly fewer $(P<0.01) \mathrm{F}$ pattern uncapacitated and significantly more $(P<0.01) \mathrm{B}$ pattern capacitated cells than seen in samples treated with crude DF alone (Fig. 5A). Thus the DF-inhibiting activity of DF-R was present in the recombinant PEBP 1 preparation, confirming the hypothesis that PEBP 1 is DF-R. The ability of PEBP 1 to inhibit DF activity decreased in a concentration-dependent manner between 7 and $2 \mathrm{nmol} / \mathrm{I}$ and activity had disappeared completely at $0.7 \mathrm{nmol} / \mathrm{l}$.

When DF-depleted suspensions were treated with DF plus $20-600 \mathrm{nmol} / \mathrm{I} \mathrm{PEBP} \mathrm{2,} \mathrm{at} \mathrm{all} \mathrm{concentrations} \mathrm{the} \mathrm{pro-}$ portions of $F$ and $B$ pattern cells were essentially those seen in cells treated with DF alone (Fig. 5B). This suggests that PEBP 2 has no DF-inhibiting activity, even when used at concentrations much higher than those at which PEBP 1 was effective. In both sets of experiments, the recombinant protein had no detectable effect when used alone on sperm suspensions (data not shown).

\section{Western blotting}

The specificity of the purified anti-peptide polyclonal antibody to PEBP 1 was confirmed by Western blotting of recombinant PEBP $1+\mathrm{His}$ and PEBP $2+\mathrm{His}$ using both an anti-His tag antibody and the anti-PEBP 1 antibody. With the former, bands of approximately the correct size $(23 \mathrm{kDa})$ were detected for both PEBP 1 and PEBP 2 samples (Fig. 6A, 6B). However, with the anti-PEBP 1 antibody, only a band for PEBP 1 was detected, despite the loading of an excess (up to 20-fold more) of PEBP 2 per lane compared with PEBP 1 (Fig. 6C); thus, there was no cross-reactivity. The anti-PEBP 1 antibody also detected a single band of $\sim 23 \mathrm{kDa}$ (Fig. 6D) in both mouse and human sperm lysates.
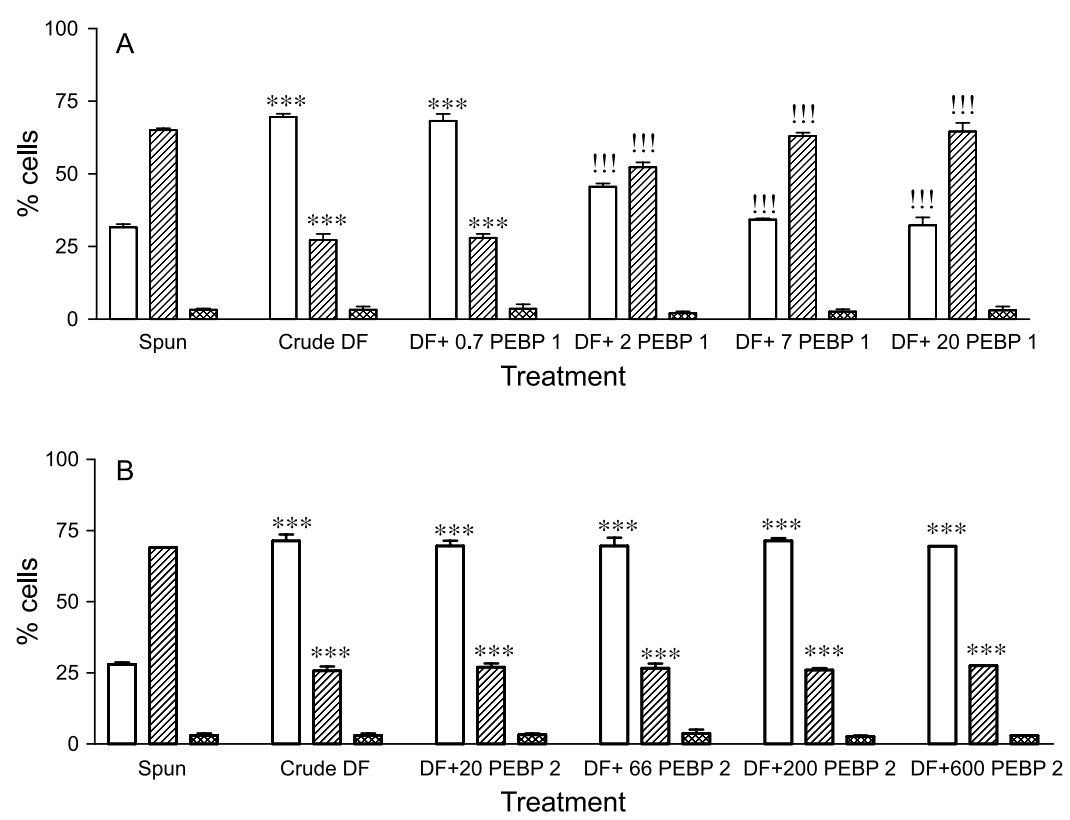

Figure 5 Biological activity of recombinant PEBP 1 and PEBP 2. (A) Mouse sperm suspensions were incubated for 25 min, centrifuged to remove endogenous DF, then incubated for $10 \mathrm{~min}$ in medium with DF plus different nanomolar concentrations of PEBP 1: spun (DF-depleted control), crude DF and DF $+0.7,2,7$ and $20 \mathrm{nmol} / \mathrm{I}$ PEBP 1 . Cells were stained with CTC, fixed and assessed for any differences in the distribution of fluorescence patterns. Data are presented as \% cells (means \pm S.E.; $n=3$ ) expressing the F pattern (open bars), the B pattern (hatched bars) and the AR pattern (cross-hatched bars) of CTC fluorescence. ${ }^{* * *} P<0.01$ compared with control suspensions and !!! $P<0.01$ compared with crude DFtreated suspensions. (B) Mouse sperm suspensions were incubated for $25 \mathrm{~min}$, centrifuged to remove endogenous DF, then incubated for $10 \mathrm{~min}$ in medium with DF plus different nanomolar concentrations of PEBP 2: spun (DF-depleted control), crude DF and DF + 20, 66, 200, and $600 \mathrm{nmol} / \mathrm{I}$ PEBP 2. Cells were stained with CTC, fixed and assessed. Data are presented as \% cells (means \pm s.E.; $n=3$ ) expressing the three different CTC patterns detailed above. ${ }^{* * *} P<0.01$ compared with control suspensions. 


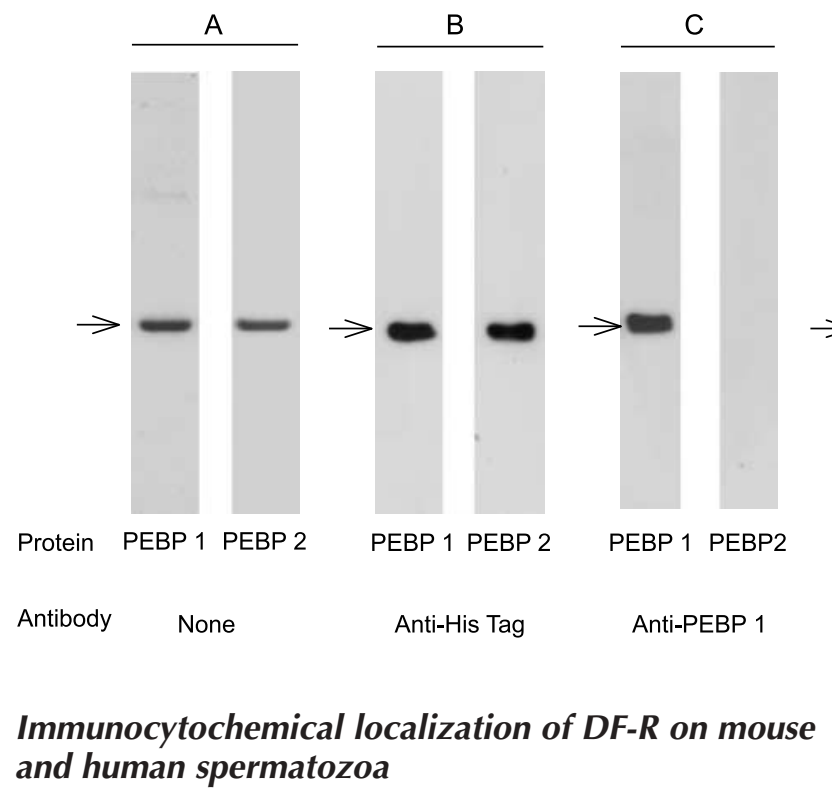

In uncapacitated mouse spermatozoa, very strong fluorescence was seen consistently on the acrosomal cap region in the head, with less intense fluorescence in the post-acrosomal region; fluorescence was observed along the whole of the flagellum but there were random regional variations in intensity from one cell to another. This fluorescence was abolished when antiserum was preincubated with the blocking peptide (data not shown). When cells were DF-depleted by centrifugation, fluorescence in the acrosomal cap region was still strong but somewhat reduced from the signal observed in uncapacitated spermatozoa; in contrast, fluorescence in the post-acrosomal region and along the whole of the flagellum was noticeably stronger than in uncapacitated spermatozoa. The re-addition of crude DF to these preparations resulted in the same fluorescence patterns observed in uncapacitated cells, indicating the reversibility of capacitation. Those same patterns were seen in naturally capacitated suspensions treated in the same way. Treatment of all

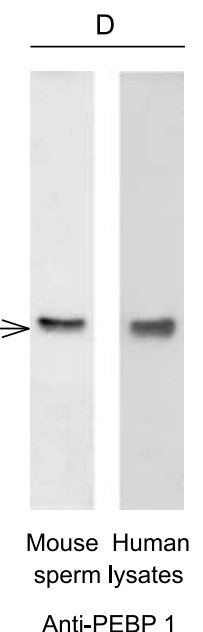

Figure 6 Specificity of polyclonal antibody to PEBP 1 determined using Western blotting. (A) These silverstained gels show the purified recombinant PEBP 1 and PEBP 2 proteins. (B) Using a mouse anti-His tag antibody, both recombinant PEBP $1+$ His (lane 1 ) and recombinant PEBP $2+$ His (lane 2 ) were detected $(\sim 23 \mathrm{kDa}$, arrow). (C) Using the polyclonal anti-PEBP 1 antibody, recombinant PEBP $1+$ His (lane 1) was detected ( $\sim 23 \mathrm{kDa}$, arrow), but recombinant PEBP $2+$ His (lane 2) was not. (D) Using the polyclonal anti-PEBP 1 antibody, a single protein of $\sim 23 \mathrm{kDa}$ (arrow) was detected in lysates of both cauda epididymal mouse spermatozoa and ejaculated human spermatozoa. suspensions with PI-PLC prior to immunocytochemistry almost completely abolished fluorescent staining. Figure 7 shows typical fluorescence patterns for uncapacitated and capacitated mouse spermatozoa, \pm DF and \pm PI-PLC treatment. At least three to four replicates were carried out for each condition. In each treatment category evaluated, $\geq 80 \%$ of cells had the pattern of fluorescence shown in Fig. 7.

In uncapacitated human spermatozoa, strong fluorescence was seen in the acrosomal region, with somewhat lesser fluorescence in the post-acrosomal region and there was noticeable fluorescence along the whole of the flagellum, although most cells had a region with reduced signal. As with mouse spermatozoa, the position of this reduced intensity varied from cell to cell. In capacitated cells, the staining in the acrosomal region was still clearly visible, but the signal was weaker and appeared to be more punctuate than that seen in uncapacitated cells; fluorescence in the post-acrosomal region was similar to that seen in uncapacitated spermatozoa. Variably intense fluorescent staining was seen along the flagellum and, again, the position of reduced intensity varied among

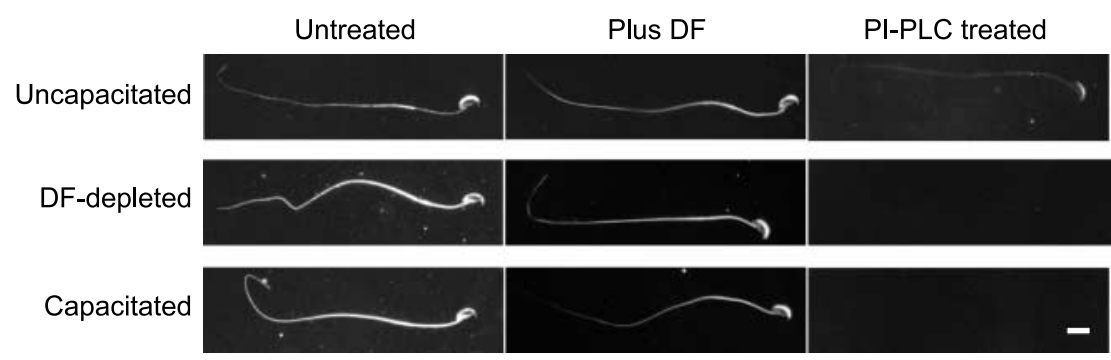

Figure 7 Immunolocalization of DF-R/PEBP 1 on mouse spermatozoa. Uncapacitated, experimentally capacitated (DF-depleted by centrifugation) and naturally capacitated suspensions were evaluated without treatment as well as following the addition of crude DF or treatment with PI-PLC to remove GPI-anchored proteins; since uncapacitated spermatozoa already have DF, the photograph in the Plus DF column simply shows a different uncapacitated cell. The intensity of fluorescence was capacitation state-dependent, with uncapacitated cells showing brightest fluorescence in the acrosomal cap region and lesser fluorescence in the post-acrosomal region and along the flagellum. In contrast, capacitated cells had noticeably brighter fluorescence in both the post-acrosomal region and the flagellum, while the signal in the acrosomal cap, although still quite strong, was less bright. The addition of crude DF to capacitated suspensions caused the staining pattern to revert to the relative intensities seen in uncapacitated cells. PI-PLC treatment prior to immunostaining resulted in almost no fluorescence. The bar represents $10 \mu \mathrm{m}$. 


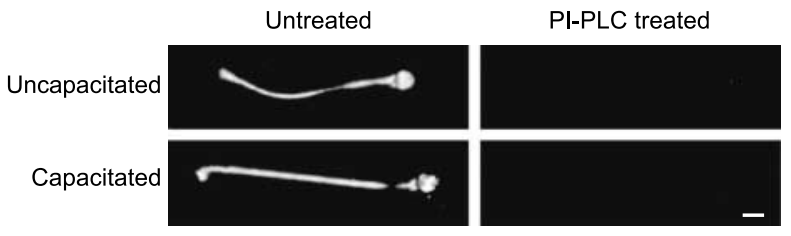

Figure 8 Immunolocalization of DF-R/PEBP 1 on human spermatozoa. Uncapacitated and capacitated human sperm suspensions were evaluated with or without treatment with PI-PLC. Fluorescence was observed in both the head and the flagellum and the signal intensity was capacitation state-dependent, as with mouse spermatozoa. Staining in the acrosomal region was very bright in uncapacitated cells but less so and more obviously punctuate in uncapacitated cells. Fluorescence was observed in the post-acrosomal region and along the flagellum in both uncapacitated and capacitated cells, with fewer differences in staining intensity than seen in mouse spermatozoa. PI-PLC treatment prior to immunostaining resulted in almost no fluorescence. The bar represents $5 \mu \mathrm{m}$.

spermatozoa. Treatment of both uncapacitated and capacitated suspensions with PI-PLC prior to immunocytochemistry resulted in non-fluorescent cells. Figure 8 shows typical fluorescence patterns for uncapacitated and capacitated human spermatozoa, \pm PI-PLC treatment. Again, three to four replicates were carried out for each condition; as with mouse spermatozoa, in each treatment category evaluated, $\geq 80 \%$ of cells had the pattern of fluorescence shown Fig. 8.

\section{Discussion}

Mouse spermatozoa have been shown to have a DF-R on the sperm surface that can be removed by treating cells with PI-PLC, suggesting that its attachment to the plasma membrane may involve a GPI anchor (Fraser 1998). Although DF is lost during capacitation, the addition of DF to capacitated cells, with consequent binding of DF to DF-R, returns them to the uncapacitated state. Thus the DF-R plays a pivotal role in the regulation of sperm function. In the present study, the sequence of DF-R, purified from mature mouse spermatozoa and shown to be biologically active, has very high homology with the sequence for PEBP 1. The peptide fragments from purified DF-R, representing $\sim 76 \%$ of the whole PEBP 1 sequence, have $100 \%$ homology when aligned with the published PEBP 1 sequence. We therefore propose that DF-R is PEBP 1.

It is now known that PEBP 1 belongs to a family of proteins, with PEBP 2 having been identified in 2002 (Hickox et al. 2002); the degree of homology between PEBP 1 and 2 is $79.7 \%$, based on the amino acid sequence. To confirm that DF-R is PEBP 1 rather than PEBP 2, we cloned the genes for both and obtained recombinant proteins, each with a His tag to facilitate purification. The purified recombinant $\mathrm{PEBP} 1$ protein was biologically active at concentrations down to $\sim 1 \mathrm{nmol} / \mathrm{l}$ : when mixed with crude DF before addition to capacitated spermatozoa, it was able to remove decapacitating activity from the DF in solution. This presumably reflects the complexing of
PEBP 1 with the DF, leaving little or no free DF to bind to endogenous DF-R present on the test cells. In contrast, purified PEBP 2, at concentrations up to $600 \mathrm{nmol} / \mathrm{l}$, had no detectable biological activity (Fig. 5B), supporting our conclusion that DF-R is PEBP 1, not PEBP 2. Given the considerable sequence similarity between these two proteins, it is perhaps surprising that PEBP 2 had no activity. One possibility could be that the presence of the His tag somehow interfered with the normal folding of PEBP 2, rendering it unable to interact with DF. However, the recombinant PEBP 1, with a His tag, had potent biological activity, as did the original sperm-derived sample used for sequencing. Using an antibody specific for PEBP 2 (unable to recognize PEBP 1 in Western blotting), Hickox et al. (2002) found PEBP 2 protein to be located in both the head and the flagellum of caput epididymal mouse spermatozoa, a distribution similar to that found for DF-R/PEBP 1 (see below). The inability of recombinant PEBP 2 to neutralize DF may indicate that it is a soluble cytosolic protein rather than an external protein like DF-R/PEBP 1 and hence may have structural differences that make it unable to function as DF-R.

In the present study, a polyclonal antibody directed against a peptide sequence at the $\mathrm{COOH}$ terminus of PEBP 1 was used both for Western blotting and immunolocalization. The antibody was specific, being able to recognize recombinant PEBP 1 but not recombinant $\mathrm{PEBP}$ 2; furthermore, it also recognized a protein of the correct size in mouse and human sperm lysates (Fig. 6). Immunocytochemical results revealed, first, that DF-R is located on both the acrosomal and post-acrosomal regions of the head and along the whole of the flagellum in both mouse and human spermatozoa and, secondly, that the intensity of fluorescent signal observed is capacitation statedependent. In uncapacitated cells, there was strong fluorescence in the acrosomal region, with weaker signals in the post-acrosomal and flagellar regions. In capacitated spermatozoa, however, the fluorescence in the acrosomal region, although still bright, was less than that seen in uncapacitated cells while the signals in the post-acrosomal and flagellar regions were generally stronger than in uncapacitated cells. However, when both naturally capacitated and DF-depleted mouse spermatozoa were evaluated before and after addition of crude DF, cells in both treatment groups initially had bright fluorescence in the acrosomal cap, post-acrosomal and flagellar regions but the addition of DF caused the fluorescence pattern to change to that seen in uncapacitated cells. Because the patterns of fluorescence are reversible, the differences probably reflect changes in epitope accessibility, due to changes in the conformation of DF-R, rather than loss or gain of protein. We would expect these alterations to have functional consequences, given earlier evidence that addition of DF to capacitated sperm suspensions rapidly causes highly fertile cells to become poorly fertile (Fraser 1984, Fraser et al. 1990). Treatment of both uncapacitated and capacitated mouse and human spermatozoa with 
PI-PLC to cleave GPI anchors prior to staining resulted in cells with essentially no fluorescent signal, thus supporting earlier evidence that DF-R is located on the external sperm surface (Fraser 1998).

It is intriguing that many different studies on mammalian tissues have reported finding proteins that appear to be PEBPs and yet these proteins differ in important characteristics, especially their cellular location. The original paper on the purification of PEBP from bovine brain described it as a basic 23 kDa cytosolic protein (Bernier \& Jolles 1984), but it appears to have homology with a number of other proteins, including several identified in various regions of the male reproductive tract. These include the mouse antigen MEP-9, a protein found in elongated spermatids in the testis and in the principal cells of the epididymal epithelium (Vierula et al. 1992), and B109, a protein isolated from the rat epididymis (Pryor et al. 1994). However, other investigations have reported that PEBP is secreted from isolated round and elongating rat spermatids (Saunders et al. 1995), is secreted into the epididymal luminal fluid (Araki et al. 1992) and is associated with the surface of mature rodent spermatozoa (e.g. rat: Jones \& Hall 1991). In somatic cells and systems, PEBP has been found in cerebrospinal fluid (Ojika et al. 2000) and it is secreted by Rat1 fibroblasts, as well as being present on the surface of those cells (Hengst et al. 2001). These latter observations are both puzzling and noteworthy because there is no obvious secretion signal in the protein's sequence. Some of these discrepancies may be due to the existence of multiple PEBP forms, the presence of which only became clear in 2002 when PEBP 2 was reported (Hickox et al. 2002). However, our evidence that DF-R/PEBP 1 is located on the sperm surface suggests that similar forms may also be present in somatic cells, thus perhaps explaining some of the inconsistencies found in the literature.

Although DF-R/PEBP 1 is released from the sperm surface by PI-PLC treatment, it may not have a GPI anchor itself. Typical GPI-anchored proteins are synthesized as precursors with cleavable hydrophobic regions at both the amino- and carboxy-terminal regions, the latter directing GPI anchoring (Mayor \& Riezman 2004). The sequence for DF-R isolated from mature spermatozoa lacked the amino terminal region, which could reflect $\mathrm{NH}_{2}$-terminal blockage but might also result from the cleavage just mentioned. However, the sequence was complete at the carboxy-terminal end, suggesting that no cleavage had occurred there. Current evidence indicates that these cleavable regions found in $\mathrm{GPI}$-anchored proteins do not have conserved sequences (Yang et al. 2004), so there is nothing with which the PEBP 1 sequence can be compared. Thus it would appear that DF-R/PEBP 1 lacks the typical structure characteristic of $\mathrm{GPI}$-anchored proteins. However, as mentioned above, the protein also lacks a classic secretion signal and yet has been shown to be secreted by both somatic and germ cells. Our results therefore suggest that DF-R/PEBP 1 works in a novel way that has yet to be defined. If DF-R itself is not GPI anchored, then it is likely to be closely associated with a GPI-linked protein. It is interesting to note that $\sim 80 \%$ of $\mathrm{GPI}$-anchored proteins can exist in multiple forms, e.g. soluble as well as anchored externally (Yang et al. 2004).

The present investigation which led us to PEBP 1 started with DF-R, a protein of unknown sequence for which we had a function, an apparent location in the post-acrosomal region and a means of attachment to a specific cell type. Although the current literature contains many speculations about possible functions of the PEBPs, the starting point of all those studies was the identification of either an mRNA or a protein of unknown function for which some role was then sought. The suggested functions for PEBP 1 include remodelling of the sperm surface (Saunders et al. 1995), a Raf kinase inhibitor (Yeung et al. 1999), a serine protease inhibitor (Hengst et al. 2001) and mediators of MAP-kinase-mediated phosphorylation (Hickox et al. 2002).

Of the potential roles ascribed to PEBP 1, remodelling of the cell surface most closely fits the current information about DF-R. Although earlier studies found a link between $\mathrm{DF} \leftrightarrow \mathrm{DF}-\mathrm{R}$ and $\mathrm{Ca}^{2+}$-ATPase activity (Adeoya-Osiguwa \& Fraser 1996), our more recent work indicates that binding of DF to spermatozoa has more wide-ranging effects, consistent with the presence of DF-R in the acrosomal cap and flagellar regions, in addition to the post-acrosomal region where $\mathrm{Ca}^{2+}$-ATPase is primarily located (Adeoya-Osiguwa \& Fraser 1996). The capacitation state-dependent alterations in the immunofluorescent signal for DF-R in both mouse and human spermatozoa (Figs 7 and 8) suggest that the conformation of DF-R itself changes with the presence/absence of DF. Consistent with that hypothesis, we have shown that the binding of DF to DF-R causes conformational changes in adenosine receptors ( $G$ protein coupled receptors; GCPRs) such that inactive stimulatory $A_{2 A}$ receptors are re-activated and active inhibitory $A_{1}$ receptors are inactivated within a few minutes of DF addition to capacitated sperm suspensions (Fraser \& Adeoya-Osiguwa 1999, Adeoya-Osiguwa \& Fraser 2002).

In the last two decades it has become clear that lipids in membranes are not distributed randomly, but are often grouped into distinct domains, commonly referred to as lipid rafts, and many proteins located in rafts have been shown to mediate signal transduction. Recent studies have demonstrated dynamic reorganization of marker proteins for sperm surface lipid rafts during capacitation in vitro (Cross 2004, van Gestel et al. 2005). Given that GPIanchored proteins are frequently associated with these cholesterol- and sphingolipid-enriched microdomains (Lai 2003), it is plausible that DF $\leftrightarrow$ DF-R could be involved in raft dynamics, causing changes in the lipid architecture of the plasma membrane and so altering the functionality of several membrane-associated proteins, including GPCRs and $\mathrm{Ca}^{2+}$-ATPase. $\mathrm{A}_{2 \mathrm{~A}}$ and $\mathrm{A}_{1}$ adenosine receptors and calcitonin receptors, like DF- $R$, are all located in the acrosomal cap region of the sperm head and along the flagellum and undergo capacitation state-dependent changes in 
function (Fraser et al. 2003). We therefore propose that DF-R/PEBP 1 plays a fundamental role in capacitation by causing alterations in the sperm plasma membrane in both the head and the flagellum.

The new and novel information about DF-R obtained in this study has raised several important issues. For example, where in the male reproductive tract is the protein produced? Although pebp 1 gene expression and protein have been detected in testicular germ cells (Vierula et al. 1992, Saunders et al. 1995), protein has also been detected in principal cells of the epididymal epithelium (Vierula et al. 1992). Using PCR we have identified the presence of mRNA for PEBP 1 in epididymal preparations (Fig. 4) but do not yet know whether the source of the mRNA is epididymal cells or spermatozoa present within the lumen of the samples used. Another key step will be purification and sequencing of DF, followed by cloning of the gene and production of specific antibodies. Then it should be possible to address a number of important questions that have been raised during our studies to date; these include the following. How is DF lost or activated during capacitation? What is the effect of DF $\leftrightarrow$ DF-R on lipid architecture? Is DF-R located in lipid rafts?

Although the existence of DFs has been acknowledged for several decades, this is the first study to provide both solid information about the molecular nature of a pivotal protein involved in capacitation/decapacitation and a plausible mechanism of action, namely alterations in membrane lipid architecture that can modify activity of membrane-associated proteins and so affect sperm function. In addition, this work has exciting potential applications both to fertility treatments, since DF retention would result in non-fertilizing spermatozoa, and to development of new contraceptive approaches, given that molecular modification of either DF or DF-R could render spermatozoa irreversibly non-fertilizing.

\section{Acknowledgements}

We thank Nicola Leeds and Steve Lynham of Proteome Sciences, Institute of Psychiatry, King's College London for carrying out the LC/MS/MS. RG is the recipient of a CASEBBSRC PhD studentship, with Pfizer Global Research and Development (Sandwich, Kent, UK) being the industrial partner for the award. The authors declare that there is no conflict of interest that would prejudice the impartiality of this scientific work.

\section{References}

Adeoya-Osiguwa SA \& Fraser LR 1996 Evidence for $\mathrm{Ca}^{2+}$-ATPase activity, stimulated by decapitation factor and calmodulin, in mouse sperm. Molecular Reproduction and Development $\mathbf{4 4}$ $111-120$.

Adeoya-Osiguwa SA \& Fraser LR 2002 Capacitation state-dependent changes in adenosine receptors and their regulation of adenylyl cyclase/cAMP. Molecular Reproduction and Development $\mathbf{6 3}$ 245-255.
Araki Y, Vierula Me, Rankin TL, Tulsiani DRP \& Orgebin-Crist M-C 1992 Isolation and characterization of a 25-kilodalton protein from mouse testis: sequence homology with a phospholipid-binding protein. Biology of Reproduction 47 832-843.

Austin CR 1952 The 'capacitation' of the mammalian sperm. Nature 170326.

Bedford JM \& Chang MC 1962 Removal of decapacitation factor from seminal plasma by high-speed centrifugation. American Journal of Physiology 202 179-181.

Bernier I \& Jolles P 1984 Purification and characterization of a basic $23 \mathrm{kDa}$ cytosolic protein from bovine brain. Biochimica et Biophysica Acta 790 174-181.

Cross NL 2004 Reorganization of lipid rafts during capacitation of human sperm. Biology of Reproduction 71 1367-1373.

DasGupta S, Mills CL \& Fraser LR 1994 A possible role for $\mathrm{Ca}^{2+}$. ATPase in human sperm capacitation. Journal of Reproduction and Fertility 102 107-116.

de Lamirande E, Leclerc P \& Gagnon C 1997 Capacitation as a regulatory event that primes spermatozoa for the acrosome reaction and fertilization. Molecular Human Reproduction 3 175-194.

Fraser LR 1984 Mouse sperm capacitation in vitro involves loss of a surface-associated inhibitory component. Journal of Reproduction and Fertility 72 373-384.

Fraser LR 1993 In vitro capacitation and fertilization. In Methods in Enzymology; Guide to Techniques in Mouse Development. Eds PM Wasserman \& ML DePamphilis 225 239-253.

Fraser LR 1998 Interactions between a decapacitation factor and mouse spermatozoa appear to involve fucose residues and a GPIanchored receptor. Molecular Reproduction and Development $\mathbf{5 1}$ 193-202.

Fraser LR \& Adeoya-Osiguwa SA 1999 Modulation of adenylyl cyclase by FPP and adenosine involves stimulatory and inhibitory adenosine receptors and G proteins. Molecular Reproduction and Development 53 459-471.

Fraser LR \& Osiguwa OO 2004 Human sperm responses to calcitonin, angiotensin II and FPP in prepared semen samples from normal donors and infertility patients. Human Reproduction 19 596-606.

Fraser LR, Harrison RAP \& Herod JE 1990 Characterization of a decapacitation factor associated with epididymal mouse spermatozoa. Journal of Reproduction and Fertility 89 135-148.

Fraser LR, Adeoya-Osiguwa SA \& Baxendale RW 2003 First messenger regulation of capacitation via G protein-coupled mechanisms: a tale of serendipity and discovery. Molecular Human Reproduction 9 739-748.

Hengst U, Albrecht H, Hess D \& Monard D 2001 The phosphatidylethanolamine-binding protein is the prototype of a novel family of serine protease inhibitors. Journal of Biological Chemistry 276 535-540.

Hickox DM, Gibbs G, Morrison JR, Sebire K, Edgar K, Hooi-Hong K, Alter K, Loveland KL, Hearn MTW, de Kretser DM \& O'Bryan MK 2002 Identification of a novel testis-specific member of the phosphatidylethanolamine binding protein family, pebp-2. Biology of Reproduction 67 917-927.

Jones R \& Hall L 1991 A $23 \mathrm{kDa}$ protein from rat sperm plasma membranes shows sequence similarity and phospholipids binding properties to a bovine brain cytosolic protein. Biochimica et Biophysica Acta 1080 78-82.

Lai EC 2003 Lipid rafts make for slippery platforms. Journal of Cell Biology 162 365-370.

Mayor S \& Riezman H 2004 Sorting GPI-anchored proteins. Nature Reviews - Molecular Cell Biology 5 110-120.

Ojika K, Mitake S, Tohdoh N, Appel SH, Otsuka Y, Katada E \& Matsukawa N 2000 Hippocampal cholinergic neurostimulating peptides (HCNP). Progress in Neurobiology $6037-83$.

Pryor JL, Xu W, Moore A, Ensrud KM \& Hamilton DW 1994 Putative rat sperm lipid-binding protein: isolation and partial 
characterization. Molecular Reproduction and Development 39 289-296.

Rakha AM, Adeoya-Osiguwa SA \& Fraser LR 2000 Purification of a mouse sperm decapacitation factor and its receptor. Journal of Reproduction and Fertility Abstract Series $\mathbf{2 6} 25$.

Saunders PTK, McKinnell C, Millar MR, Gaughan J, Turner KJ, Jégou B, Syed V \& Sharpe RM 1995 Phosphatidylethanolamine binding protein is an abundant secretory product of haploid testicular germ cells in the rat. Molecular and Cellular Endocrinology $107221-230$.

Snedecor GW \& Cochran WG 1980 In Statistical Methods, edn 7. Ames: lowa State University Press.

van Gestel RA, Brewis IA, Ashton PR, Helms JB, Brouwers JF \& Gadella BM 2005 Capacitation dependent concentration of lipid rafts in the apical ridge head area of porcine sperm cells. Molecular Human Reproduction 11 [In Press].

Vierula ME, Araki Y, Rankin TL, Tulsiani DR \& Orgebin-Crist MC 1992 Immunolocalization of a 25-kilodalton protein in mouse testis and epididymis. Biology of Reproduction 47 844-856.
Yang J, Tiong J, Dennard M \& Jefferies WA 2004 Deletion of the GPI pre-anchor sequence in human p97 - a general approach for generating the soluble form of GPI-linked proteins. Protein Expression and Purification 34 28-48.

Yeung K, Seitz T, Li S, Janosch P, McFerran B, Kaiser C, Fee F, Katsanakis KD, Rose DW, Mischak H, Sedivy JM \& Kolch W 1999 Suppression of Raf- 1 kinase activity and MAP kinase signalling by RKIP. Nature 401 173-177.

Zenewicz LA, Wei, Goldfine H \& Shen H 2005 Phosphatidylinositol-specific phospholipase $\mathrm{C}$ of Bacillus anthracis downmodulates the immune response. Journal of Immunology $\mathbf{1 7 4}$ 8011-8016.

Received 10 May 2005

First decision 23 June 2005

Revised manuscript received 23 June 2005

Accepted 4 July 2005 